\title{
113 CISH GENE-KNOCKOUT ANTI-CD70-CAR NK CELLS DEMONSTRATE POTENT ANTI-TUMOR ACTIVITY AGAINST SOLID TUMOR CELL LINES AND PROVIDE PARTIAL RESISTANCE TO TUMOR MICROENVIRONMENT INHIBITION
}

Chao Guo*, Yanying Fan, Alexander Aronov, Luxuan Buren, Ming-Hong Xie, Ivan Chan, Sasha Lazetic, James Trager. NKARTA THERAPEUTICS, South San Francisco, CA, USA

Background Peripheral blood natural killer (NK) cells are attractive candidates for adoptive cell therapy. NK cells possess innate ability for tumor cell killing and are also amenable to genomic engineering for enhanced functions. Moreover, NK cells possess an inherent capacity for allogeneic, off-the-shelf therapy since, unlike $\mathrm{T}$ cells, they are neither HLA-restricted nor known to cause graft-versus-host disease. Cytokine inducible $\mathrm{SH} 2$-containing protein $(\mathrm{CISH})$ is a negative regulator of interleukin 15 (IL-15) signaling in natural killer (NK) cells. Here we show the potential application of CISH gene-knockout CAR NK cells targeting CD70 and expressing a membrane-bound form of IL-15. CD70 is an antigen that is aberrantly expressed in a variety of malignant settings, including renal cell carcinoma (RCC), while its expression in normal tissues is restricted to a subset of lymphoid cell types.

Methods To target CD70 on RCC cells, we generated CD70CAR NK cells with CISH deletion. Using the CRISPR/Cas9 system, we knocked out CISH expression in isolated peripheral blood NK cells from healthy donors. Since CD70 expression is present on activated NK cells, we also targeted CD70 for CRISPR knockout to avoid fratricide. We then expanded these edited NK cells by using IL-2 and stimulation using NKSTIM, a modified K562 stimulatory cell line expressing membrane-bound form of IL-15 (mbIL-15) and 4-1BBL. IL-12 and IL-18 were added during expansion to drive memory-like NK cell differentiation. We transduced the expanded NK cells to express engineered CD70-targeted CAR and mbIL-15. We assessed CAR expression, NK cell persistence, and NK cell activity against RCC target cells using end-point cytotoxicity assays and IncuCyte.

Results CISH gene-knockout CD70-CAR NK cells could be produced efficiently and exhibited extended persistence in culture. After engineering and expansion, CD70-CAR transduction efficiency was 60-80\%. CD70-CAR NK cells displayed potent cytotoxicity against CD70-expressing renal cancer derived cell lines. Interestingly, cytotoxicity assays demonstrated that CISH gene-knockout CD70-CAR NK cells were partially resistant to TGFß and adenosine inhibition of cytotoxicity. Furthermore, CISH gene-knockout CD70-CAR NK cells maintained their activity during prolonged culture.

Conclusions In summary, we show CISH gene-knockout CD70-CAR NK cells demonstrate potent anti-tumor activity against relevant solid tumor cell lines and partially provide resistance to tumor microenvironment inhibition. These data support the further exploration of CISH gene-knockout CD70 CAR NK cells for clinical application.

http://dx.doi.org/10.1136/jitc-2021-SITC2021.113 